\title{
Association between Circulating Protein C Levels and Incident Dementia: The Atherosclerosis Risk in Communities Study
}

\author{
Adrienne Tin ${ }^{a, b}$ Keenan A. Walker ${ }^{c}$ Jan Bressler ${ }^{d} \quad$ B. Gwen Windham ${ }^{a}$ \\ Michael Griswold ${ }^{a}$ Kevin Sullivan ${ }^{a}$ Aozhou Wu ${ }^{b}$ Rebecca Gottesman ${ }^{\mathrm{e}}$ \\ Myriam Fornage $^{d}$ Josef Coresh ${ }^{b}$ A. Richey Sharrett ${ }^{b}$ Aaron R. Folsom ${ }^{f}$ \\ Thomas H. Mosley ${ }^{\mathrm{a}}$ \\ aDepartment of Medicine, University of Mississippi Medical Center, Jackson, MS, USA; ${ }^{\mathrm{b}}$ Department of Epidemiology, \\ Johns Hopkins Bloomberg School of Public Health, Baltimore, MD, USA; ' Laboratory of Behavioral Neuroscience, \\ Intramural Research Program, National Institute on Aging, Baltimore, MD, USA; dHuman Genetics Center, School of \\ Public Health, University of Texas Health Science Center at Houston, Houston, TX, USA; eDepartment of Neurology, \\ Johns Hopkins University, Baltimore, MD, USA; fDivision of Epidemiology and Community Health, University of \\ Minnesota, Minneapolis, MN, USA
}

\section{Keywords}

Protein C D Dementia $\cdot$ Hemostasis

\begin{abstract}
Introduction: Hemostasis depends on the delicate balance between coagulants and anticoagulants. Higher levels of circulating coagulants have been associated with higher risk of cerebral infarctions and dementia. In contrast, higher levels of circulating protein $C$, an endogenous anticoagulant, have been associated with lower risk of cerebral infarctions, and the association between protein $C$ levels and the risk of dementia is unknown. The goal of this study was to evaluate the association of circulating protein $C$ levels in midlife and late life with incident dementia. Methods: Circulating protein $C$ levels were measured using blood samples collected at the midlife baseline (1987-1989) and the late-life baseline (2011-2013) among 14,462 and 3,614 participants, respectively, in the Atherosclerosis Risk in Communities study. Protein $C$ levels were measured using enzyme-linked immunosorbent assay at midlife and a modified aptamer-based assay
\end{abstract}

at late life. Participants were followed up to 2013 from midlife and up to 2017 from late life. Incident dementia was ascertained during the follow-up periods using in-person cognitive and functional assessment, informant interviews, and International Classification of Diseases codes at hospitalization discharge and on death certificates. Cause-specific Cox regression models were used to evaluate the association between quintiles of circulating protein $C$ and incident dementia. Results: From midlife (mean age of 54), 1,389 incident dementia events were observed over a median follow-up of 23 years. From late life (mean age of 75), 353 incident dementia events were observed over a median follow-up of 4.9 years. At both midlife and late life, circulating protein $C$ had an inverse association with incident dementia after adjusting for demographic, vascular, and hemostatic risk factors, incident stroke as time-dependent covariate, and incorporating stabilized weights based on propensity scores (quintile 5 vs. quintile 1 as the reference, midlife hazard ratio 0.80 , $95 \%$ confidence interval $0.66-0.96, p$ value for trend 0.04 ; late-life hazard ratio $0.84,95 \%$ confidence interval: $0.55-$ 1.28, $p$ value for trend 0.04). Discussion/Conclusion: Circu- karger@karger.com www.karger.com/ned

Karger $\stackrel{\text { ' }}{5}$

BOPEN ACCESS
(C) 2021 The Author(s)

Published by S. Karger AG, Basel

This is an Open Access article licensed under the Creative Commons Attribution-NonCommercial-4.0 International License (CC BY-NC) (http://www.karger.com/Services/OpenAccessLicense), applicable to the online version of the article only. Usage and distribution for commercial purposes requires written permission.
Correspondence to:

Adrienne Tin, atin@umc.edu 
Fig. 1. Overview of the study design. The associations between protein $\mathrm{C}$ levels and incident dementia were assessed using 2 follow-up periods with nonoverlapping sets of cases. The midlife baseline was 1987-1989 (visit 1) with follow-up period up to the end of 2013. The late-life baseline was 2011-2013 (visit 5) with follow-up period up to the end of 2017. ELISA, enzymelinked immunosorbent assay.

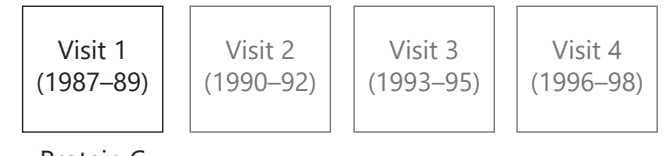

Protein C

(ELISA)

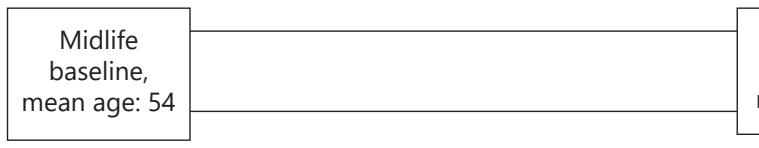

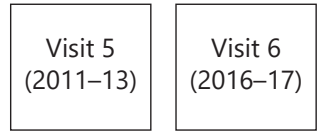

Protein C

(SomaScan)

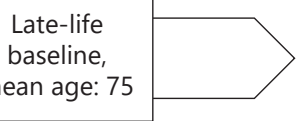

lating protein $\mathrm{C}$ has an inverse association with incident dementia independent of established risk factors, including stroke. Our results suggest studying anticoagulants in addition to coagulants can increase our understanding on the relationship between hemostasis and dementia.

(C) 2021 The Author(s)

Published by S. Karger AG, Basel

\section{Introduction}

Hemostasis, which maintains blood fluidity and stops bleeding in the event of vascular injury, depends on the delicate balance between coagulants and anticoagulants [1]. In population-based studies, higher levels of circulating coagulants have been associated with higher risk of cerebral infarctions, cognitive decline, and dementia [25]. In contrast, higher levels of circulating protein $C$ have been associated with lower risk of cerebral infarctions, a risk factor for dementia $[4,6]$. Among patients with atrial fibrillation, oral anticoagulant use has been associated with lower risk for dementia [7, 8]. Little is known about the association between endogenous anticoagulant levels and the risk of dementia in the wider population of middle aged and older adults.

Protein $\mathrm{C}$ is an endogenous anticoagulant and, upon activation, deactivates 2 coagulants, factor $\mathrm{V}$ and factor VIII, whose levels have a positive association with cognitive decline and the risk of dementia [2,9]. Higher circulating protein $\mathrm{C}$ levels have been associated with lower risk of ischemic stroke, an established risk factor for dementia [10]. However, many patients with dementia do not have stroke preceding dementia but have evidence of cerebral small vessel disease as revealed in magnetic resonance imaging (MRI) scans [11]. Studying the association between circulating protein $C$ levels and incident dementia may provide insight into the early role of the balance of hemostasis in dementia risk. We evaluated the potential etiological relationship of pro- tein $\mathrm{C}$ levels in midlife and late life with incident dementia using cause-specific Cox regression models [12].

\section{Materials and Methods}

\section{Overview of Study Design}

The Atherosclerosis Risk in Communities (ARIC) study is a longitudinal cohort study of 15,972 adults from 4 communities (Washington County, MD, USA; Forsyth County, NC, USA; northwestern suburbs of Minneapolis, MN, USA; and Jackson, MS, USA) with Jackson by design enrolling self-reported Black participants only. Since enrollment (visit 1, 1987-1989), 7 visits have been conducted.

Our primary analysis assessed the associations of circulating protein C levels in midlife and late life with incident dementia using 2 follow-up periods with nonoverlapping sets of incident dementia cases (Fig. 1). The midlife baseline was 1987-1989 (visit 1, mean age: 54 years). The association analysis from midlife included 14,462 participants who were followed up to the end of visit 5 (December 31, 2013). The exclusions at midlife were 103 participants with numbers in self-reported race and center combinations too small for analysis (self-reported race that was not white or black, or self-reported as black in Washington County or Minneapolis), 1 with missing incident dementia status, 275 with missing protein $\mathrm{C}$ measures, 587 with missing values for $A P O E_{\varepsilon} 4$ genotype, 289 with missing values for other baseline covariates, and 75 with anticoagulant use (warfarin for all). Details are reported in online suppl. Figure 1; for all online suppl. material, see www.karger.com/ doi/10.1159/000516287. The late-life baseline was 2011-2013 (visit 5 , mean age: 75 years). The association from late life included 3,614 of the 6,538 participants who attended the late-life baseline visit, and participants were followed up to December 31, 2017, when incident stroke information was available for all participants from the ARIC cohort surveillance [13]. The exclusions at late life were: 42 participants with self-reported race that was not white or black, or self-reported as black in Washington County or Minneapolis, 341 with prevalent dementia, 609 missing dementia status, 1,081 without protein $\mathrm{C}$ measures, 185 with missing values for $A P O E_{\varepsilon} 4$ genotype, 430 with missing values for other baseline covariates, and 236 with anticoagulant use (warfarin use: 218 , other anticoagulants: 18). Details are reported in online suppl. Figure 2. Given that warfarin is known to suppress protein C activity [14], 
participants who reported the use of warfarin at the midlife and late-life baselines were excluded. Participants who reported the use of other anticoagulants were also excluded because the small number of users made it infeasible to include the use of other anticoagulants as a covariate.

\section{Standard Protocol Approvals, Registrations, and Patient}

Consents

This study was approved by the Institutional Review Board of each ARIC participating site: University of North Carolina at Chapel Hill, Chapel Hill, NC, USA; Wake Forest University, Winston-Salem, NC, USA; Johns Hopkins University, Baltimore, MD, USA; University of Minnesota, Minneapolis, MN, USA; and University of Mississippi Medical Center, Jackson, MS, USA. All participants provided written informed consent at each study visit, and proxies provided consent for participants who were determined to lack capacity.

\section{Measurement of Circulating Protein C Levels}

The blood samples used for the assay of protein $\mathrm{C}$ were drawn from the antecubital vein after an 8-h fast using a citrated plasma tube in midlife and an EDTA plasma tube in late life. Samples were processed according to a standardized protocol and shipped on dry ice to the ARIC central laboratory for storage at $-70^{\circ} \mathrm{C}$ or $-80^{\circ} \mathrm{C}$. At the midlife baseline, circulating protein $\mathrm{C}$ antigen levels were measured within a few weeks of blood draw using enzymelinked immunosorbent assay [15-18]. The coefficient of variation from blind duplicates was $4-5 \%$ [17]. The short-term reliability coefficients obtained from repeated testing of individuals over several weeks were 0.56 for protein C, 0.72 for fibrinogen, and 0.68 for von Willebrand factor [19]. At the late-life baseline, the relative concentration of protein $\mathrm{C}$ in relative fluorescence units was measured after 5-7 years of storage using SomaScan version 4, a modified aptamer-based assay [20,21]. Based on binding-specificity tests performed by the manufacturer, the modified aptamers that targeted protein $\mathrm{C}$ did not bind to the related proteins (factor VII, factor IX or factor X). The specificity of the modified aptamertargeting protein $\mathrm{C}$ was also confirmed by data-dependent analysis mass spectrometry [22]. The protein $\mathrm{C}$ measures were normalized at the modified aptamer, plate, and sample levels based on the manufacturer standards and finally adjusted using the protein measures of a pool of healthy controls [23]. Based on blind duplicates ( $n=197$ pairs), the coefficient of variation of protein $C$ at the late-life baseline was 4.6\% [24]. The Spearman correlation between the midlife and late-life protein $\mathrm{C}$ measures was 0.18 .

\section{Incident Dementia Ascertainment}

We followed up participants from enrollment (1987-1989, visit 1 ) to the end of visit 6 (December 31,2017). Dementia status was determined by an expert committee that included physicians and neuropsychologists. The methods for dementia ascertainment in the ARIC study have been reported previously [25]. Briefly, the ascertainment was based on detailed cognitive and functional assessment (online suppl. Table 1) at ARIC visits 5 (2011-2013) and 6 (2016-2017), cognitive tests conducted at visits 2 (1990-1992) and 4 (1996-1998), and informant interviews. Participants who did not attend visit 5 were contacted by phone and administered the modified Telephone Interview for Cognitive Status (TICS) [26]. TICS scores were education-adjusted [25]. Informant interviews were sought for participants who could not be contacted for visits 5 or 6 . During visit 5 , informant interviews used the Clinical
Dementia Rating and the Functional Activities Questionnaire. After visit 5, telephone interviews were sought for all participants semiannually between study visits using the Six-Item Screener (SIS) scores and for informant interviews using the Alzheimer's Dementia 8-Item Informant Questionnaire [27]. Finally, dementia cases were also ascertained using ICD-9 dementia codes at hospitalization discharge and on death certificates obtained by ARIC cohort surveillance.

Dementia date was first set as the earliest of either the hospitalization date with an ICD-9 code for dementia, death date if a dementia code was listed on the death certificate, date of telephone communication with the participant or proxy with indication of dementia, or date of the first visit when dementia was indicated. Dementia onset date ascertained from informant interviews, hospitalization, and death certificates was reassigned as 6 months earlier to account for the expected lag in the reporting of the event. Participants who were classified as not having dementia were censored at their last study contact date or the date of death collected by ARIC cohort surveillance.

\section{Measurement of Other Variables}

Race, education levels (<high school, high school graduate, or vacation school, and at least some college, graduate, or professional school), and current smoking status were self-reported. Body mass index (BMI) was calculated using height and weight measured at study visit. Prevalent diabetes mellitus was defined as having a fasting glucose level $\geq 126 \mathrm{mg} / \mathrm{dL}$, nonfasting glucose level $\geq 200 \mathrm{mg} / \mathrm{dL}$, self-reported diabetes medication use, or self-reported physician diagnosis of diabetes. Hypertension was defined as systolic blood pressure $\geq 140 \mathrm{~mm} \mathrm{Hg}$, diastolic blood pressure $\geq 90$ $\mathrm{mm} \mathrm{Hg}$, or self-reported use of antihypertension medications. Estimated glomerular filtration rate (eGFR) was calculated using the Chronic Kidney Disease Epidemiology Collaboration equation [28] with calibrated and standardized serum creatinine [29]. Anticoagulant use was determined based on the medication bottles that the participants provided at study visit. Platelet count was measured by Coulter counters. At the midlife baseline, factor VIII activity (factor VIIIc) was measured by the coagulation test, fibrinogen by the thrombin-time titration method, and von Willebrand factor antigen by enzyme-linked immunosorbent assay. At the late-life baseline, factor VIII, fibrinogen, and von Willebrand factor were measured by the SomaScan assay. Global cognition Z scores were calculated by averaging the $Z$ scores of 3 tests: the Delayed Word Recall Test, the Digit Symbol Substitution Test from the Wechsler Adult Intelligence Scale-Revised, and the Word Fluency Test $[30,31]$. Prevalent stroke at the midlife baseline was based on self-report. Subsequent stroke events were based on hospital records from local hospital surveillance and annual telephone interview with ARIC participants. Stroke events were classified by a combination of computer algorithm and physician review [32]. Atrial fibrillation events were ascertained based on electrocardiogram during study visits, ICD codes for hospitalization discharge, and on death certificate [33]. Genotyping of the 2 single-nucleotide polymorphisms (rs429358, rs7412) that define the $A P O E_{\varepsilon} 4$ genotypes was performed using the TaqMan assay (Thermo Fisher Scientific, Waltham MA, USA).

\section{Statistical Analysis}

Baseline characteristics were compared across quintiles of circulating protein $\mathrm{C}$ levels using the $\chi^{2}$ test for categorical variables 


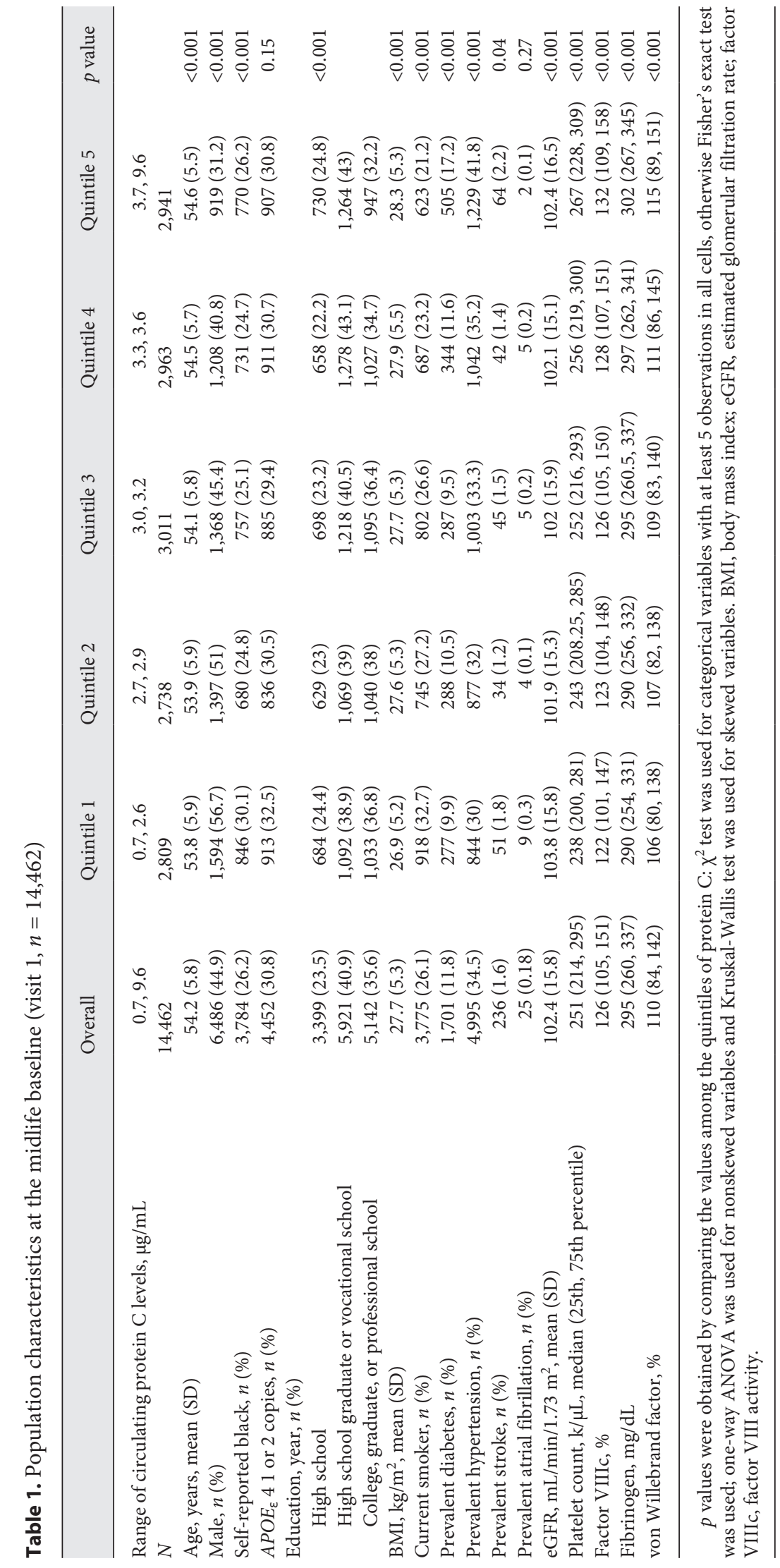


with $\geq 5$ observations in all cells and the Fisher's exact test otherwise, one-way ANOVA for nonskewed continuous variables, and Kruskal-Wallis test for skewed continuous variables. We performed cause-specific analysis using Cox regression models, which are appropriate for etiological investigation [12] to evaluate the association of circulating protein $\mathrm{C}$ levels at midlife and late life with incident dementia. Protein $\mathrm{C}$ levels at both midlife and latelife baselines were categorized in quintiles. Covariates were established demographic, genetic, or vascular risk factors for dementia $[34,35]$ or novel risk factors that may be confounders in the relationship between protein C levels and dementia: eGFR [36], 3 coagulation factors (factor VIII, fibrinogen, and von Willebrand factor) [3], and platelet count $[37,38]$.

Our primary analysis consists of 5 cause-specific Cox regression models. At the midlife baseline, the covariates of model 1 included demographics (age, race-center, sex, education levels) and $A P O E_{\square} 4$ carrier status. Model 2 added vascular risk factors (diabetes, hypertension, BMI, current smoking, and prevalent stroke). Model 3 added coagulants (factor VIIIc, fibrinogen, and von Willebrand factor) and platelet count. Model 4 added incident stroke as a time-varying covariate. Model 5 added the inverse of propensity scores as weights to further control for potential confounding. The propensity scores represent the conditional probabilities of belonging to one of the quintiles of protein $\mathrm{C}$ given the covariates $[39,40]$. Stabilized weights were calculated as a ratio with the numerator being the density of the quintiles of protein $\mathrm{C}$ and the denominator being the propensity scores generated using all covariates in model 4 . To assess whether these stabilized weights indeed balanced the covariates across quintiles of protein $\mathrm{C}$, we conducted weighted regression analysis using each covariate as the outcome and quintiles of protein $\mathrm{C}$ as predictors incorporating the stabilized weights. The results showed that the covariates were largely independent of protein $\mathrm{C}$ after incorporating the stabilized weights (online suppl. Table 2).

At the late-life baseline, the 5 models were essentially the same as those defined for the midlife baseline except as noted here. First, the values of BMI, current smoking status, prevalent stroke, diabetes and hypertension status, eGFR, factor VIII, von Willebrand factor, and platelet count were obtained at the late-life baseline. Second, models 2-5 included global cognition $\mathrm{Z}$ score, which was not available at the midlife baseline. Last, models 3-5 did not include fibrinogen because the modified aptamer-targeted fibrinogen did not pass quality control for binding specificity. Similar to the results of the midlife baseline, the covariates were largely independent of protein $\mathrm{C}$ after incorporating the stabilized weights (online suppl. Table 3).

We conducted 2 secondary analyses. Given that the protein $\mathrm{C}$ levels might be more related to vascular risk factors of dementia, and the $A P O E_{\varepsilon} 4$ genotype is more specific for Alzheimer's disease, the first secondary analysis assessed the association between protein $\mathrm{C}$ levels and incident dementia excluding participants who were $A P O E_{\varepsilon} 4$ homozygotes (model 6) and $A P O E_{\varepsilon} 4$ carriers (model 7). Model 6 had the same covariates as model 5, and model 7 removed $A P O E_{\varepsilon} 4$ carrier status as a covariate. Both models 6 and 7 were evaluated incorporating stabilized weights based on propensity scores as used in model 5 . The other secondary analysis was conducted at the midlife baseline to explore whether only very low levels of protein $\mathrm{C}$ within quintile 1 were associated with higher risk of dementia given that compared with quintile 1, all higher quintiles (from 2 to 5 ) had similar hazard ratios (HRs). This anal- ysis modeled protein $\mathrm{C}$ levels using cubic spline with knots at the 10 th and 20th percentiles and included the same covariates and propensity score-based weights as in model 5 . This analysis was not performed for the late-life baseline given that compared to quintile 1 , quintile 2 had slightly higher risk estimate and quintiles 3-5 had lower risk estimates for dementia.

In all analyses, to reduce skewedness and potential influence of outliers, a $\log 2$ transformation was applied to factor VIII, fibrinogen, von Willebrand factor, and platelet count followed by winsorization at the 1st and 99th percentile. The proportional hazards assumption was assessed by inspecting the Schoenfeld residual plot and testing whether the slope of the time-dependent coefficient across time was zero [41]. The $p$ value for trend was obtained by using the 5 quintiles of protein $C$ as a continuous variable with values from 1 to 5 . The $W$ ald test with a degree of freedom of 4 was used to assess the joint effects of all 5 quintiles. All analyses were conducted using R 4.0.2. The stabilized weights based on propensity scores were generated using the $\mathrm{R}$ ipw package [40].

\section{Data Availability Statement}

ARIC data from visit 1 to visit 5 are available through the Biologic Specimen and Data Repository Information Coordinating Center (BioLINCC). Data that are not yet available through BioLINCC are available upon request through the ARIC Coordinating Center at the University of North Carolina.

\section{Results}

At the midlife baseline (mean age: 54 years, $45 \%$ men, $26 \%$ black overall), the median protein C level was $3.1 \mu \mathrm{g} /$ $\mathrm{mL}$ (25th, 75th percentile: 2.7, 3.5). Higher quintiles of circulating protein $\mathrm{C}$ levels had lower proportions of men, current smokers, black participants, as well as higher BMI, and proportions of prevalent diabetes and hypertension. The proportions of participants with prevalent atrial fibrillation and $A P O E_{\varepsilon} 4$ allele were similar across the quintiles (Table 1).

After a median follow-up of 23 years, we observed 1,389 incident dementia events. The primary data sources for dementia diagnoses were in-person cognition and functional assessment (22.1\%), the Clinical Dementia Rating and/or the Functional Activities Questionnaire (38.7\%), ICD codes at hospitalization discharge (26.9\%), TICS (6.7\%), and ICD codes on death certificates $(5.5 \%$, online suppl. Table 4). The curves of the crude cumulative incidence by quintiles of protein C levels from the midlife baseline were close together (online suppl. Fig. 3). After adjusting for demographic factors (age, sex, race-center, and education levels) and $A P O E_{\varepsilon} 4$ carrier status, there was a weak inverse association between protein $C$ levels and incident dementia (model 1, quintile 1 as reference, quintile 5 adjusted $\mathrm{HR}, 0.82,95 \%$ confidence interval [CI]: $0.69-0.97, p$ value for trend 0.14 , Wald test $p$ value 
Table 2. Association between circulating protein C levels in midlife and incident dementia $(n=14,462)$

\begin{tabular}{llllllll}
\hline Model & Quintile 1 & Quintile 2 & Quintile 3 & Quintile 4 & Quintile 5 & $\begin{array}{c}p \text { value } \\
\text { for trend } \begin{array}{l}\text { Wald test } \\
p \text { value }\end{array}\end{array}$ \\
\hline Model 1 & Reference & $0.76(0.64,0.91)$ & $0.87(0.73,1.02)$ & $0.84(0.71,1.00)$ & $0.82(0.69,0.97)$ & 0.14 & 0.05 \\
Model 2 & Reference & $0.75(0.63,0.90)$ & $0.87(0.73,1.03)$ & $0.82(0.69,0.98)$ & $0.77(0.64,0.91)$ & 0.03 & 0.01 \\
Model 3 & Reference & $0.74(0.62,0.89)$ & $0.84(0.71,1.00)$ & $0.80(0.67,0.95)$ & $0.74(0.62,0.88)$ & 0.01 & 0.004 \\
Model 4 & Reference & $0.73(0.61,0.88)$ & $0.85(0.71,1.00)$ & $0.81(0.68,0.96)$ & $0.77(0.64,0.92)$ & 0.04 & 0.01 \\
Model 5 & Reference & $0.75(0.61,0.91)$ & $0.88(0.73,1.06)$ & $0.83(0.69,0.99)$ & $0.80(0.66,0.96)$ & 0.04 & 0.04 \\
\hline
\end{tabular}

Covariates for each model. Model 1: age, sex, race-center, education levels, $\mathrm{APOE}_{\varepsilon} 4$ carrier status. Model 2: model $1+$ vascular factors (BMI, current smoking status, prevalent stroke, diabetes and hypertension status, eGFR). Model 3: model $2+$ coagulants (factor VIIIc, fibrinogen, von Willebrand factor) + platelet count. Model 4: model 3 + incident stoke as time-varying covariate. Model 5: model 4 incorporating stabilized weights based on the inverse of propensity scores. $p$ value for trend was obtained by using the 5 quintiles of protein $\mathrm{C}$ as a continuous variable with values from 1 to 5 . Wald test $p$ values were obtained by testing the joint effects of all 5 quartiles. $p$ value for proportional hazards assumption for all protein $\mathrm{C}$ quintile variables $>0.10$ for all models. BMI, body mass index; eGFR, estimated glomerular filtration rate; factor VIIIc, factor VIII activity.

for the joint effects of all quintiles: 0.05, Table 2). After adjusting for vascular and hemostatic risk factors, the inverse association between protein $\mathrm{C}$ levels and incident dementia became stronger (model 3 , quintile 5 adjusted HR, $0.74,95 \%$ CI: $0.62-0.88$, $p$ value for trend 0.01 , Wald test $p$ value: 0.004 , Table 2 ). This stronger inverse association might be partly due to the inclusion of some vascular risk factors of dementia that had positive univariate relationship with protein $\mathrm{C}$ levels, such as BMI and diabetes reported above. After adjusting for additional covariates and incident stroke as a time-varying covariate (model 4), higher levels of protein $\mathrm{C}$ were associated with lower risk for dementia (quintile 5 adjusted HR, 0.77, 95\% CI: $0.64-0.92$, $p$ value for trend 0.04 , Wald test $p$ value 0.01 , Table 2). Results were similar in model 5 incorporating the stabilized weights based on the propensity scores (quintile 5 adjusted HR, 0.80, 95\% CI: 0.66-0.96, $p$ value for trend 0.04 , Wald's test $p$ value 0.04 ). The HRs for quintiles $2-5$ were similar. In the secondary analysis excluding $A P O E_{\varepsilon} 4$ homozygotes and heterozygotes (models 6 and 7), the HR estimates were similarly to those from model 5 (online suppl. Table 5). We did not observe a monotonic dose-response pattern. The secondary analysis exploring the shape of association within quintile 1 using a cubic spline with knots at the 10th and 20th percentiles revealed an inverse dose-response pattern within quintile 1 (cubic spline term overall $p$ value 0.004 ), that is, the risk for dementia was higher as the levels of protein C became lower (Fig. 2).

At the late-life baseline (mean age: 75 years, $41.3 \%$ men, $17.5 \%$ black overall), similar to the midlife baseline, higher quintiles of circulating protein $\mathrm{C}$ levels had lower

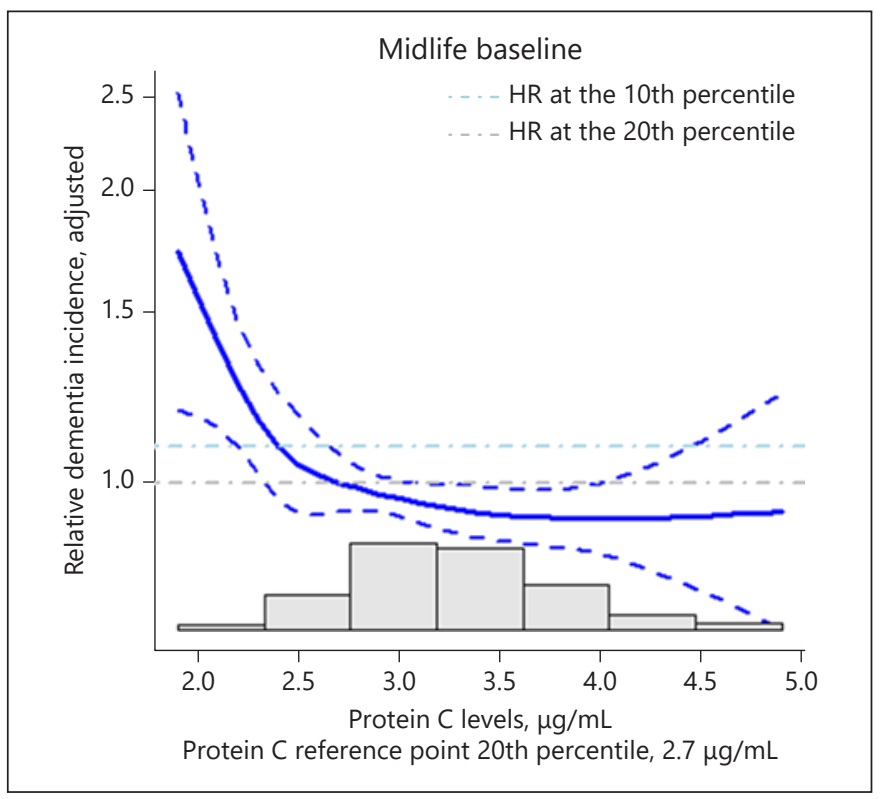

Fig. 2. At the midlife baseline, within quintile 1 , an inverse doseresponse relation between protein $\mathrm{C}$ levels and dementia risk was apparent when protein $\mathrm{Clevels}$ were modeled as a cubic spline with knots at the 10th and 20th percentiles using the same covariates and stabilized weights based on propensity score as in model 5 ( $p$ value of the cubic spline 0.004 ). The gray bars in the lower part of the plot is the histogram of protein $\mathrm{C}$ levels. HR, hazard ratio.

proportions of men and black participants. The proportions of participants with prevalent atrial fibrillation and $A P O E_{\varepsilon} 4$ allele were also similar across the quintiles. In contrast to the midlife baseline, higher quintiles of circulating protein $\mathrm{C}$ levels had lower BMI and proportions of 
Table 3. Association between circulating protein C levels in late-life and incident dementia $(n=3,614)$

\begin{tabular}{llllllll}
\hline Model & Quintile 1 & Quintile 2 & Quintile 3 & Quintile 4 & Quintile 5 & $\begin{array}{c}p \text { value } \\
\text { for trend }\end{array}$ & $\begin{array}{c}\text { Wald test } \\
p \text { value }\end{array}$ \\
\hline Model 1 & Reference & $1.07(0.80,1.44)$ & $0.69(0.50,0.96)$ & $0.66(0.46,0.94)$ & $0.79(0.55,1.13)$ & 0.01 \\
Model 2 & Reference & $1.21(0.89,1.64)$ & $0.78(0.56,1.08)$ & $0.71(0.49,1.03)$ & $0.92(0.63,1.34)$ & 0.07 & 0.01 \\
Model 3 & Reference & $1.21(0.89,1.63)$ & $0.77(0.55,1.07)$ & $0.70(0.49,1.02)$ & $0.92(0.63,1.35)$ & 0.07 & 0.02 \\
Model 4 & Reference & $1.20(0.89,1.62)$ & $0.78(0.56,1.08)$ & $0.71(0.49,1.02)$ & $0.93(0.64,1.36)$ & 0.08 & 0.02 \\
Model 5 & Reference & $1.26(0.92,1.72)$ & $0.78(0.56,1.10)$ & $0.73(0.50,1.07)$ & $0.84(0.55,1.28)$ & 0.04 & 0.02 \\
\hline
\end{tabular}

Covariates for each model. Model 1: age, sex, race-center, education levels, $A P O E_{\varepsilon} 4$ carrier status. Model 2: model $1+$ vascular factors (BMI, current smoking status, prevalent stroke, diabetes and hypertension status, eGFR). Model 3: model $2+$ coagulants (factor VIII, von Willebrand factor) + platelet count. Model 4: model 3 + incident stoke as time-varying covariate. Model 5: model 4 incorporating stabilized weights based on the inverse of propensity scores. $p$ value for trend was obtained by using the 5 quintiles of protein $\mathrm{C}$ as a continuous variable with values from 1 to 5 . Wald test $p$ values were obtained by testing the joint effects of all 5 quartiles. $p$ value for proportional hazards assumption for each quintile variable $>0.1$ for all. BMI, body mass index; eGFR, estimated glomerular filtration rate.

prevalent diabetes and hypertension (online suppl. Table 6). After a median follow-up of 4.9 years, we observed 353 incident dementia events. The primary data sources for dementia diagnoses were in-person cognition and functional assessments (37.4\%), Alzheimer's Dementia 8-Item Informant Questionnaire (35.4\%), Six-Item Screener (6.8\%), and ICD codes at hospitalization discharge (19.3\%) and on death certificates (1.1\%, online suppl. Table 7). In contrast to the midlife baseline, the curves of the crude cumulative incidence by quintiles of protein $\mathrm{Clev}$ els at late life showed a separation pattern reflecting the inverse association between protein $\mathrm{C}$ levels and incident dementia (online suppl. Fig. 4). The results from the 5 multivariate models did not differ appreciably due to covariate adjustment (Table 3). In all model tested, the overall associations between quintiles of protein $\mathrm{C}$ and incident dementia were significant (Wald test $p$ value for the joint effect of all quintiles $\leq 0.02$ ). The HRs of the quintiles had a wide CI, and quintiles 3-5 had lower HR estimates compared with quintile 1. For example, in model 5 when the stabilized weights based on propensity scores were incorporated: quintile 1 as reference, quintile 3 adjusted HR, 0.78, 95\% CI: 0.56-1.10; quintile 4 adjusted HR, 0.73, 95\% CI: 0.50-1.07; quintile 5 adjusted HR, 0.84, 95\% CI: $0.55-1.28$; $p$ value for trend 0.04 ). In the secondary analysis excluding $A P O E_{\varepsilon} 4$ homozygotes and heterozygotes, the inverse association between protein $C$ levels and incident dementia seemed to be slightly stronger in model 7 (quintile 1 as reference, quintile 5 adjusted HR 0.62, 95\% CI: $0.37,1.04, p$ trend: 0.01 , online suppl. Table $5)$. Similar to the association in midlife, we did not observe a monotonic dose-response pattern.

\section{Discussion/Conclusion}

In this study of a community-based cohort with measures of circulating protein $\mathrm{C}$ levels in midlife and latelife, cause-specific Cox regression models showed that protein $\mathrm{C}$ levels and incident dementia had an inverse association independent of stroke and coagulants, including factor VIII, fibrinogen, and von Willebrand factor. These results suggest more research on the balance between coagulants and anticoagulants in hemostasis may yield insight on the pathophysiology of dementia.

In the coagulation cascade, protein $\mathrm{C}$, upon activation, can inactivate factor VIII resulting in the lower production of fibrin downstream [42]. In addition, activated protein C reduces the activity of plasminogen activator inhibitor. This function enhances the production of plasmin, which degrades fibrin clots [43]. Therefore, adequate levels of protein $\mathrm{C}$ are critical for avoiding stasis and maintaining blood fluidity. Protein C levels have had an inverse association with incident stroke, an established risk factor for dementia $[10,44]$. In this study, the association between protein $\mathrm{C}$ and dementia was independent of stroke. Subclinical vascular injuries, such as brain infarcts, may be among the links underlying this independent inverse association between protein $\mathrm{C}$ and dementia risk. Among participants in the ARIC Brain MRI study, which were stroke free at the time of the MRI examination, higher levels of circulating protein $\mathrm{C}$ and lower levels of fibrinogen at the midlife baseline were associated with lower odds for subclinical brain infarcts 6 years later [4]. Subclinical brain infarcts have been shown to be detectable in about $20 \%$ of older adults without stroke and have been associated with 
higher risk for dementia in population-based studies $[6,45$, 46]. These results suggest the inverse association between protein $\mathrm{C}$ levels and incident dementia reported in the present study may not be mediated by stroke, and the balance between anticoagulants and coagulants in hemostasis may be important in the maintenance of vascular health in the brain for the prevention of dementia.

The inverse association between protein $\mathrm{C}$ and incident dementia is consistent with the protective association between anticoagulant treatment and incident dementia among patients with atrial fibrillation [7, 8, 47]. While atrial fibrillation is an established risk factor for dementia, the underlying mechanism is still unclear [48]. Dysrhythmia in atrial fibrillation is considered a cause of thromboembolism, which could lead to ischemic stroke or other vascular defects in the brain and thus increases the risk of dementia [49]. In addition, atrial fibrillation could also be a marker of the Virchow's triad for thromboembolism: a hypercoagulable state, stasis, and endothelial injury, which are common among older adults $[48,49]$. The results of the present study suggest that levels of endogenous anticoagulant levels may be relevant for the study of dementia pathophysiology in a wider population.

This study has several strengths. We demonstrated consistent inverse associations of circulating protein $\mathrm{C}$ levels with incident dementia in midlife and late life using 2 follow-up periods with nonoverlapping cases in a large community-based cohort including white and black participants. The dementia assessment was rigorous combining in-person and informant interviews with cohort surveillance. Some limitations warrant mentioning. At the midlife baseline, dementia assessment was not available. We assumed all participants were free of dementia at this baseline given that the age range at this baseline was $45-$ 64. Specific forms of dementia diagnosis, such as vascular dementia and Alzheimer's disease, were not available in the ARIC study. Therefore, we could not evaluate the association between protein $\mathrm{C}$ levels and specific forms of dementia. Measures of protein S, a cofactor of protein C, were not available to inform whether the effects of protein $\mathrm{C}$ might have been affected by this cofactor. Protein C measured at midlife had higher within-person variability than other hemostatic factors [19]. We could have underestimated the true association due to this measurement variability. Given that the protein $\mathrm{C}$ levels at the midlife baseline were absolute measures and at the late-life baseline were relative measures, we could not directly compare the range of the protein $\mathrm{C}$ values or the changes between these 2 baselines. While the inverse associations between protein $\mathrm{C}$ levels and the risk for dementia were consistent between the midlife and the late-life baselines, the sample size was smaller at the late-life baseline resulting in larger CIs in the comparisons between quintiles. Finally, given that this is an observational study with relative quantification of protein $\mathrm{C}$ levels at the late-life baseline, our data could not provide precise estimates of protein Clevels that could be protective for dementia risk.

In summary, we have showed that circulating protein $\mathrm{C}$ levels have an inverse association with incident dementia. Our results suggest investigating anticoagulants in conjunction with coagulants in hemostasis may yield insight into the pathophysiology of dementia, potentially leading to its prevention.

\section{Acknowledgements}

The authors thank the staff and participants of the ARIC study for their important contributions.

\section{Statement of Ethics}

Study participants gave their written informed consent and that the study protocol was approved by the institute's committee on human research.

\section{Conflict of Interest Statement}

The authors have no conflicts of interest to declare.

\section{Funding Sources}

The Atherosclerosis Risk in Communities Study is carried out as a collaborative study supported by National Heart, Lung, and Blood Institute contracts (HHSN268201700001I, HHSN268201700002I, HHSN268201700003I, HHSN268201700005I, HHSN268201700004I). Neurocognitive data are collected by U01 2U01HL096812, 2U01HL096814, 2U01HL096899, 2U01HL096902, and 2U01HL096917 from the NIH (NHLBI, NINDS, NIA, and NIDCD), and with previous brain MRI examinations funded by R01-HL70825 from the NHLBI.

\section{Author Contributions}

A.T. had the original idea for the study and designed the study. A.T. performed the statistical analysis and wrote the first draft. R.G., A.R.F., J.C., and T.H.M. had major role in the acquisition of the data. All authors critically reviewed and wrote the final version. All authors approved the final version of the manuscript. A.T. and T.H.M. had full access to all the data in the study and had final responsibility for the decision to submit for publication. 


\section{References}

1 Fisher MJ. Brain regulation of thrombosis and hemostasis: from theory to practice. Stroke. 2013;44(11):3275-85.

2 Alexander KS, Zakai NA, Gillett S, McClure LA, Wadley V, Unverzagt F, et al. ABO blood type, factor VIII, and incident cognitive impairment in the REGARDS cohort. Neurology. 2014;83(14):1271-6.

3 Walker KA, Gottesman RF, Wu A, Knopman DS, Gross AL, Mosley TH, et al. Systemic inflammation during midlife and cognitive change over 20 years: the ARIC Study. Neurology. 2019;92(11):e1256-67.

4 Knuiman MW, Folsom AR, Chambless LE, Liao D, Wu KK. Association of hemostatic variables with MRI-detected cerebral abnormalities: the atherosclerosis risk in communities study. Neuroepidemiology. 2001;20(2): 96-104.

5 Quinn TJ, Gallacher J, Deary IJ, Lowe GDO, Fenton C, Stott DJ. Association between circulating hemostatic measures and dementia or cognitive impairment: systematic review and meta-analyzes. J Thromb Haemost. 2011; 9(8):1475-82.

6 Sigurdsson S, Aspelund T, Kjartansson O, Gudmundsson Elias F, Jonsdottir Maria K, Eiriksdottir G, et al. Incidence of brain infarcts, cognitive change, and risk of dementia in the general population: the AGES-Reykjavik Study (Age Gene/Environment Susceptibility-Reykjavik Study). Stroke. 2017;48(9): 2353-60.

7 Madhavan M, Hu TY, Gersh BJ, Roger VL, Killian J, Weston SA, et al. Efficacy of warfarin anticoagulation and incident dementia in a community-based cohort of atrial fibrillation. Mayo Clin Proc. 2018;93(2):145-54.

8 Chen N, Lutsey Pamela L, MacLehose Richard F, Claxton J'Neka S, Norby Faye L, Chamberlain Alanna M, et al. Association of oral anticoagulant type with risk of dementia among patients with nonvalvular atrial fibrillation. J Am Heart Assoc. 2018;7(21): e009561.

9 Gallacher J, Bayer A, Lowe G, Fish M, Pickering J, Pedro S, et al. Is sticky blood bad for the brain?: hemostatic and inflammatory systems and dementia in the Caerphilly Prospective Study. Arterioscler Thromb Vasc Biol. 2010; 30(3):599-604.

10 Kuzma E, Lourida I, Moore SF, Levine DA, Ukoumunne OC, Llewellyn DJ. Stroke and dementia risk: a systematic review and metaanalysis. Alzheimers Dement. 2018;14(11): 1416-26.

11 Lopez OL, Kuller LH, Becker JT, Jagust WJ, DeKosky ST, Fitzpatrick A, et al. Classification of vascular dementia in the cardiovascular health Study Cognition Study. Neurology. 2005;64(9):1539-47.

12 Austin PC, Lee DS, Fine JP. Introduction to the analysis of survival data in the presence of competing risks. Circulation. 2016;133(6): $601-9$.
13 White AD, Folsom AR, Chambless LE, Sharret AR, Yang K, Conwill D, et al. Community surveillance of coronary heart disease in the atherosclerosis risk in communities (ARIC) Study: methods and initial two years' experience. J Clin Epidemiol. 1996;49(2):223-33.

14 Esmon CT, Vigano-D’Angelo S, D’Angelo A, Comp PC. Anticoagulation proteins $\mathrm{C}$ and $\mathrm{S}$. Adv Exp Med Biol. 1987;214:47-54.

15 Papp AC, Hatzakis H, Bracey A, Wu KK. ARIC hemostasis study-I. Development of a blood collection and processing system suitable for multicenter hemostatic studies. Thromb Haemost. 1989;61(1):15-9.

16 Wu KK, Papp AC, Patsch W, Rock R, Eckfeldt J, Sharrett R. ARIC hemostasis study-II. Organizational plan and feasibility study. Thromb Haemost. 1990;64(4):521-5.

17 Chambless LE, McMahon R, Finch A, Sorlie P, Heiss G, Lyles R, et al. ARIC hemostasis study-III. Quality control. Atherosclerosis risk in communities. Thromb Haemost. 1993; 70(4):588-94.

18 Nguyen ND, Ghaddar H, Stinson V, Chambless LE, Wu KK. ARIC hemostasis study-IV. Intraindividual variability and reliability of hemostatic factors. The atherosclerosis risk in communities (ARIC). Thromb Haemost. 1995;73(2):256-60.

19 Chambless LE, McMahon R, Wu K, Folsom A, Finch A, Shen YL. Short-term intraindividual variability in hemostasis factors. the aric study. atherosclerosis risk in communities intraindividual variability study. Ann Epidemiol. 1992;2(5):723-33.

20 Gold L, Ayers D, Bertino J, Bock C, Bock A, Brody EN, et al. Aptamer-based multiplexed proteomic technology for biomarker discovery. PLoS One. 2010;5(12):e15004.

21 Ostroff R, Foreman T, Keeney TR, Stratford S, Walker JJ, Zichi D. The stability of the circulating human proteome to variations in sample collection and handling procedures measured with an aptamer-based proteomics array. J Proteomics. 2010;73(3):649-66.

22 Emilsson V, Ilkov M, Lamb JR, Finkel N, Gudmundsson EF, Pitts R, et al. Co-regulatory networks of human serum proteins link genetics to disease. Science. 2018;361:769.

23 Candia J, Cheung F, Kotliarov Y, Fantoni G, Sellers B, Griesman T, et al. Assessment of variability in the SOMAscan assay. Sci Rep. 2017;7(1):14248.

24 Bland JM, Altman DG. Measurement error proportional to the mean. BMJ. 1996; 313(7049): 106.

25 Knopman DS, Gottesman RF, Sharrett AR, Wruck LM, Windham BG, Coker L, et al. Mild Cognitive impairment and dementia prevalence: the atherosclerosis risk in Communities Neurocognitive Study (ARIC-NCS). Alzheimers Dement. 2016;2:1-11.

26 Knopman DS, Roberts RO, Geda YE, Pankratz VS, Christianson TJ, Petersen RC, et al. Validation of the telephone interview for cog- nitive status-modified in subjects with normal cognition, mild cognitive impairment, or dementia. Neuroepidemiology. 2010;34(1): $34-42$.

27 Carpenter CR, DesPain B, Keeling TN, Shah $M$, Rothenberger $M$. The six-item screener and $\mathrm{AD} 8$ for the detection of cognitive impairment in geriatric emergency department patients. Ann Emerg Med. 2011;57(6):653-61.

28 Levey AS, Stevens LA, Schmid CH, Zhang YL, Castro AF 3rd, Feldman HI, et al. A new equation to estimate glomerular filtration rate. Ann Intern Med. 2009;150(9):604-12.

29 Selvin E, Manzi J, Stevens LA, Van Lente F, Lacher DA, Levey AS, et al. Calibration of serum creatinine in the National Health and Nutrition Examination Surveys (NHANES) 1988-1994, 1999-2004. Am J Kidney Dis. 2007;50(6):918-26

30 Knopman DS, Ryberg S. A verbal memory test with high predictive accuracy for dementia of the Alzheimer type. Arch Neurol. 1989;46(2): 141-5.

31 Rawlings AM, Sharrett AR, Schneider AL, Coresh J, Albert M, Couper D, et al. Diabetes in midlife and cognitive change over 20 years: a cohort study. Ann Intern Med. 2014; 161(11):785-93.

32 Rosamond WD, Folsom AR, Chambless LE, Wang $\mathrm{CH}, \mathrm{McGovern} \mathrm{PG}$, Howard G, et al. Stroke incidence and survival among middleaged adults: 9-year follow-up of the atherosclerosis risk in communities (ARIC) cohort. Stroke. 1999;30(4):736-43.

33 Chen LY, Norby FL, Gottesman RF, Mosley TH, Soliman EZ, Agarwal SK, et al. Association of atrial fibrillation with cognitive decline and dementia over 20 years: the ARIC-NCS (Atherosclerosis Risk in Communities Neurocognitive Study). J Am Heart Assoc. 2018; 7(6):e007301.

34 Baumgart M, Snyder HM, Carrillo MC, Fazio $\mathrm{S}$, Kim H, Johns H. Summary of the evidence on modifiable risk factors for cognitive decline and dementia: a population-based perspective. Alzheimers Dement. 2015;11(6): 718-26.

35 Livingston G, Sommerlad A, Orgeta V, Costafreda SG, Huntley J, Ames D, et al. Dementia prevention, intervention, and care. Lancet. 2017;390(10113):2673-734.

36 Christensson A, Ash JA, DeLisle RK, Gaspar FW, Ostroff R, Grubb A, et al. The impact of the glomerular filtration rate on the human plasma proteome. Proteomics Clin Appl. 2018 May;12(3):e1700067.

37 White TC, Berny MA, Tucker EI, Urbanus RT, de Groot PG, Fernández JA, et al. Protein $C$ supports platelet binding and activation under flow: role of glycoprotein Ib and apolipoprotein E receptor 2. J Thromb Haemost. 2008;6(6):995-1002.

38 Catricala S, Torti M, Ricevuti G. Alzheimer disease and platelets: how's that relevant. Immun Ageing. 2012;9(1):20. 
39 van der Wal WM, Prins M, Lumbreras B, Geskus RB. A simple G-computation algorithm to quantify the causal effect of a secondary illness on the progression of a chronic disease. Stat Med. 2009;28(18):2325-37.

40 van der Wal WM, Geskus RB. ipw: an R package for inverse probability weighting. J Stat Softw. 2011;1(13):2011.

41 Grambsch PM, Therneau TM. Proportional hazards tests and diagnostics based on weighted residuals. Biometrika. 1994;81(3): 515-26.

42 Hoffman M, Monroe DM 3rd. A cell-based model of hemostasis. Thromb Haemost. 2001;85(6):958-65.
43 van Hinsbergh VW, Bertina RM, van Wijngaarden A, van Tilburg NH, Emeis JJ, Haverkate $\mathrm{F}$. Activated protein $\mathrm{C}$ decreases plasminogen activator-inhibitor activity in endothelial cell-conditioned medium. Blood. 1985; 65(2):444-51.

44 Folsom AR, Ohira T, Yamagishi K, Cushman $\mathrm{M}$. Low protein $\mathrm{C}$ and incidence of ischemic stroke and coronary heart disease: the Atherosclerosis Risk in Communities (ARIC) Study. J Thromb Haemost. 2009;7(11):1774-8.

45 Vermeer SE, Prins ND, den Heijer T, Hofman A, Koudstaal PJ, Breteler MM. Silent brain infarcts and the risk of dementia and cognitive decline. N Engl J Med. 2003;348(13):1215-22.
46 Prabhakaran S, Wright CB, Yoshita M, Delapaz R, Brown T, DeCarli C, et al. Prevalence and determinants of subclinical brain infarction: the Northern Manhattan Study. Neurology. 2008;70(6):425-30.

47 Field TS, Weijs B, Curcio A, Giustozzi M, Sudikas S, Katholing A, et al. Incident atrial fibrillation, dementia and the role of anticoagulation: a Population-Based Cohort Study. Thromb Haemost. 2019;119(6):981-91.

48 Kamel H, Okin PM, Elkind MS, Iadecola C. Atrial fibrillation and mechanisms of stroke: time for a new model. Stroke. 2016;47(3): 895-900.

49 Watson T, Shantsila E, Lip GY. Mechanisms of thrombogenesis in atrial fibrillation: Virchow's triad revisited. Lancet. 2009;373(9658): $155-66$. 